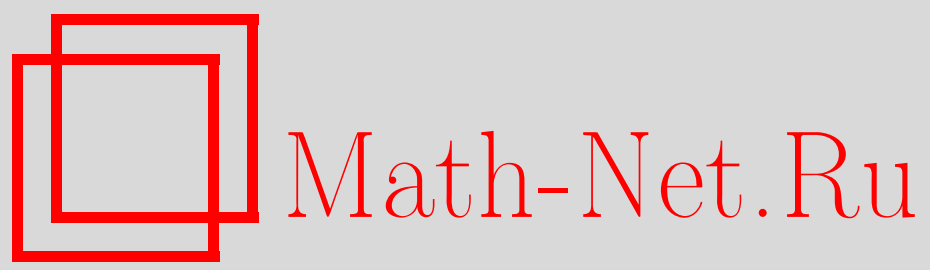

А. Урысон, Космические лучи и исследование космоса, Квант, 2020, номер 7, 2-8

DOI: https://doi.org/10.4213/kvant20200701

Использование Общероссийского математического портала Math-Net.Ru подразумевает, что вы прочитали и согласны с пользовательским соглашением http://www . mathnet.ru/rus/agreement

Параметры загрузки:

IP : 54.198 .55 .26

26 апреля 2023 г., 14:08:18

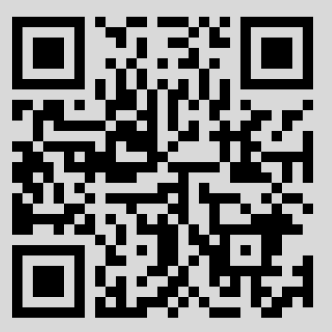




\section{Космические лучи и исследование космоса}

\section{А. УРЫСОН}

\section{Что такое космические лучи}

Космические лучи - это элементарные частицы (протоны, электроны) и ядра атомов, которые летят к нам из космоса. В космических лучах были зарегистрированы частицы очень больших энергий - до 48 Дж. На ускорителях такие энергии недостижимы. Нужно сказать, что физики обычно используют для указания энергии не джоуль, а другую единицу - электронвольт (эВ). В этих единицах максимальная энергия, зарегистриро-

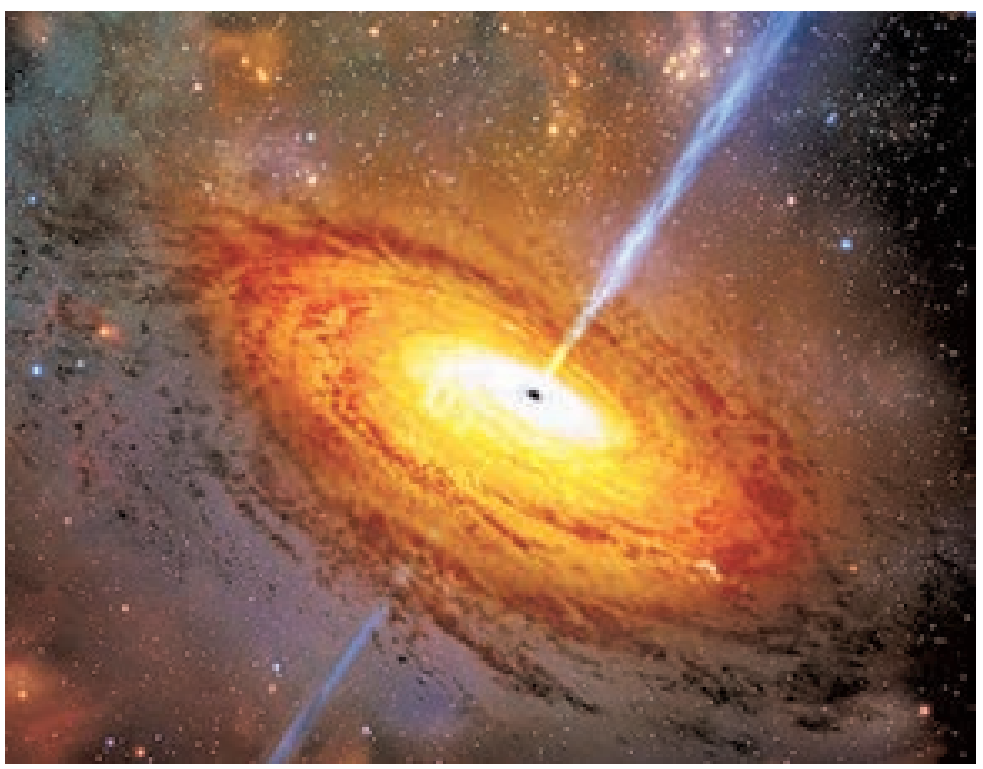
ванная у частиц космических лучей, равна $3 \cdot 10^{20}$ эВ. А весь диапазон энергий космических частиц чрезвычайно широк: от $10^{6}$ эВ до $3 \cdot 10^{20}$ эВ. Космические лучи даже самых низких энергий летят с гигантскими скоростями, почти со скоростью света.

Откуда берутся космические лучи?

Частицы с энергией ниже $2 \cdot 10^{10}$ эВ поставляются Солнцем, и они называются солнечными космическими лучами. Частицы более высоких энергий, вплоть до $10^{17}-10^{18}$ эВ, рождаются в Галактике во взрывах сверхновых, и они называются галактическими космическими лучами. Частицы еще больших энергий, выше $10^{19}$ эВ, называются космическими лучами ультравысоких энергий. В этом названии нет информации о происхождении частиц, а только указывается их энергетический диапазон. Причина в том, что происхожде-

DOI: https://doi.org/10.4213/kvant20200701 ние космических лучей ультравысоких энергий до сих пор не выяснено.

Как исследуют космические лучи ультравысоких энергий? Их регистрируют детекторы на наземных установках, а затем при помощи пакетов специальных программ определяются энергии частиц, направления их прихода и другие характеристики. Теперь, дополнительно к этому способу, данные о космических лучах получают, используя приборы, размещенные на борту спутников.

Где же находятся те природные ускорители, в которых частицы космических лучей получают такую огромную энергию? После многолетних исследований выяснилось следующее. Во-первых, природные ускорители расположены вне нашей галактики Млечный Путь, а во-вторых, их можно исследовать астрономическими методами. 


\section{За пределами Млечного Пути. Активные ядра галактик}

Млечный Путь - лишь одна из многочисленных галактик нашей Вселенной. Галактики различаются по внешнему виду, по размеру, по интенсивности звездообразования, по соотношению старых и молодых звезд. По этим признакам их и классифицируют астрономы. Но галактики различаются еще и характеристиками своей центральной части (это тоже важный признак для классификации галактик). Центральная часть отличается от других областей галактики повышенной концентрацией звезд и межзвездного вещества - газа и пыли. Эта часть галактики компактна. Ее называют ядром галактики. Причиной сгущения вещества в ядре галактики является гравитация. Однако не у всех галактик есть ядра. Их нет у небольших по массе галактик, так как там недостаточно гравитации для сгущения вещества в центре.

В большинстве галактик основная часть энергии излучается звездами, и излучение галактического ядра - это тоже излучение составляющих его звезд. Такие галактические ядра называются стационарными или неактивными.

Но существует небольшое количество галактик, в которых из ядра вырывается огромный поток энергии, как если бы в центре галактики светило сто миллионов или даже сто миллиардов Солнц. Излучение из ядра переменное - оно резко уменьшается и потом резко возрастает, причем за короткий промежуток времени (часы, месяцы, годы). При этом энергия ядра излучается в различных диапазонах - радио-, рентгеновском, ультрафиолетовом, инфракрасном и гамма-диапазоне. Из ядра выбрасываются порции плазмы, вблизи него быстро движутся облака газа. Эти процессы невозможно объяснить высокой плотностью звезд и межзвездного вещества. Такие ядра называются активными или нестационарными.

Галактик с активными ядрами немного, примерно 1\% от общего количества галактик. Их подразделяют на несколько типов в зависимости от того, как проявляется активность ядра. Например, самым мощным излучением обладают квазары, галактики с мощным радиоизлучением из ядра называются радиогалактиками.

Мы не будем касаться классификации активных ядер, так как она не важна для нашего исследования. А суть его состоит в следующем. Поскольку в активных галактических ядрах выделяется огромное количество энергии, то там, возможно, и протекают процессы, в которых космические лучи приобретают ультравысокие энергии. Вылетев из области, где частицы ускорялись, космические лучи покидают пределы своей галактики и, преодолев огромные расстояния в космосе, достигают Земли. Можно ли исследовать активные ядра, регистрируя на Земле эти космические лучи, пусть частицы и проделали гигантский путь в космосе?

В этом исследовании важно ответить на такие вопросы: откуда в активных галактических ядрах берется колоссальная энергия; как там ускоряются частицы; что происходит с частицами, когда они летят в межгалактическом пространстве.

\section{Что является источником энергии активного галактического ядра}

В настоящее время доминирует гипотеза, что активность ядра обусловлена сверхмассивной черной дырой в центре галактики.

Черная дыра - это объект с такой сильной гравитацией, что из него не может вырваться даже свет. Как это может быть? Для того чтобы преодолеть силу притяжения небесного тела, тела должны иметь вторую космическую скорость. Для черных дыр вторая космическая скорость больше скорости света. Но в природе ничто не движется быстрее света, поэтому из черных дыр ничего не выходит наружу. Так как из них не выходит даже свет, черные дыры - темные, они невидимы для наблюдателя.

На больших расстояниях от черной дыры ее притяжение очень мало и не отличается от притяжения звезд. Лишь начиная с какого-то расстояния, все, попавшее в гра- 
витационное поле черной дыры, «сваливается» в нее и уже не может выйти наружу. Граница, отделяющая ту область вокруг черной дыры, из которой ничего не возвращается, называется горизонтом черной дыры, или горизонтом событий. Никакие сигналы или тела, находящиеся внутри горизонта событий, не могут выйти из черной дыры.

Если масса $M$ черной дыры составляет не менее $10^{5}$ масс Солнца $M_{\odot}$, то такая дыра называется сверхмассивной. В ядрах галактик наблюдаются признаки черных дыр с $M \approx\left(10^{5}-10^{11}\right) \cdot M_{\odot}$.

\section{Аккреционный диск вокруг сверхмассивной черной дыры}

Под действием тяготения на черную дыру падает вещество из окружающего пространства. Это вещество составляют звезды, оказавшиеся слишком близко от черной дыры и затянутые ее гравитационным полем, газ и пыль из межзвездной среды, а также вещество, захваченное с поверхности соседних звезд.

В гравитационном поле черной дыры потенциальная энергия падающего вещества преобразуется в кинетическую, и вещество разгоняется до огромных скоростей, сравнимых со скоростью света. Из-за того что звезды обращаются по орбитам, вещество падает на черную дыру не вертикально, а закручиваясь, образуя вокруг черной дыры плотный и горячий диск так называемый аккреционный диск.

Слои газа в диске движутся вокруг центра в одном направлении, но с разными скоростями - чем ближе к центру, тем выше скорости. Поэтому между слоями газа возникает трение, и оно превращает кинетическую энергию газа в тепло. В результате диск разогревается до такой высокой температуры, что светится в радио-, инфракрасном и оптическом диапазонах, в рентгеновском свете и гаммалучах.

Газ в аккреционном диске не только вращается, но и медленно перемещается по радиусу к центру. Скорость этого движения зависит от характеристик диска массы и температуры. В некоторых аккре- ционных дисках радиальное перемещение газа к центру приводит к тому, что по оси диска перпендикулярно его плоскости выбрасываются массы вещества. Они формируются в узкие прерывистые струи, или джеты (от английского jet-cmpyя). Сверхмассивная черная дыра с аккреционным диском и джетами изображена на рисунке в начале статьи.

У аккреционного диска есть магнитное поле. Оно образует магнитосферу вокруг черной дыры. О том, почему в аккреционном диске существует магнитное поле, рассказывается в приложении «Магнитное поле аккреционного диска».

\section{Ускорение частиц вблизи сверхмассивных черных дыр}

Вблизи сверхмассивных черных дыр имеется три зоны, где может происходить ускорение частиц. Это магнитосфера черной дыры, аккреционный диск и джет. В каждой зоне частицы ускоряются своими способами. О них рассказывается в приложении «Способы ускорения частиц вблизи сверхмассивных черных дыр».

А можно ли определить, в какой зоне были ускорены космические лучи?

Ускоренные частицы имеют разную энергию. Определив количество частиц, имеющих одинаковую энергию, и построив график зависимости числа частиц от энергии, мы получим энергетический спектр частиц - число частиц $d N$ с энергией $\delta$ в единичном интервале энергии $d \varnothing$. Энергетический спектр можно получить и аналитически (в виде формулы). Теоретический анализ показывает, что в каждой зоне ускорения энергетический спектр частиц будет разным.

Когда частицы ускоряются в джете, их энергетический спектр описывается формулой $\frac{d N}{d \delta} \propto \delta^{-\alpha}$, показатель степени $\alpha \approx(2,2-2,5)$. Спектр такого вида называется степенным (или экспоненциальным). Чем выше энергия, тем быстрее уменьшается число частиц с ростом энергии.

Когда частицы ускоряются в аккреционном диске, их энергетический спектр тоже 


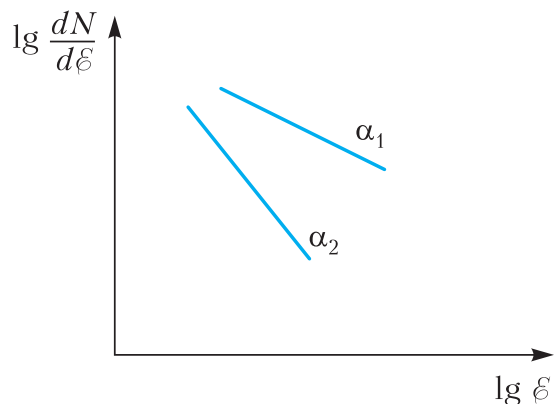

Рис. 1. Схематический график исходного степенного спектра космических лучей в логарифмическом масштабе. Показаны две кривые с разными показателями спектра

степенной, но показатель степени другой: $\alpha \approx(0-2,1)$. Показатель степени 0 - это вариант ускорения, когда при любых энергиях число частиц в одинаковых интервалах энергии одинаковое.

Степенные энергетические спектры с разными показателями $\alpha_{1}$ и $\alpha_{2}\left(\alpha_{1}<\alpha_{2}\right)$ схематически показаны на рисунке 1.

Когда частицы ускоряются в магнитосфере черной дыры, почти все они набирают примерно одну и ту же энергию. Такой энергетический спектр называется моноэнергетическим, он показан на рисунке 2.

Итак, космические лучи, ускоренные в разных зонах, имеют разные энергетические спектры.

Самый простой ход мысли дальше мог бы быть таким. Измерим энергетический спектр космических лучей на Земле и сравним его с теоретическими спектрами (изображенными на рисунках 1, 2). Выяс-

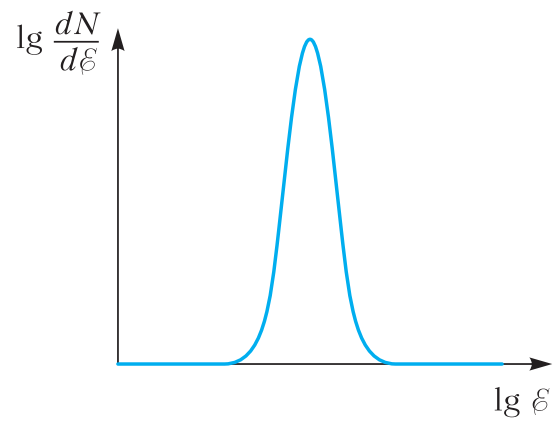

Рис. 2. Схематический график исходного моноэнергетического спектра космических лучей в логарифмическом масштабе ним, с каким из них совпадает измеренный спектр, и найдем, где ускорялись космические лучи. Так мы «заглянем» в окрестности черной дыры и увидим, как там ускоряются частицы.

Но этот простой ход мысли неверный, и вот почему.

\section{Что происходит с космическими лучами, когда они летят во Вселенной}

Покинув свой «ускоритель» - активное ядро галактики, космические лучи затем вылетают из своей галактики. Те из частиц, которые достигают Земли, преодолевают расстояния в десятки и сотни миллионов световых лет. Это огромные расстояния. Их проходят за десятки и сотни миллионов лет фотоны, а они летят с самой большой скоростью в природе.

Что происходит с космическими лучами, когда они летят во Вселенной? Галактики распределены во Вселенной редко, на больших расстояниях друг от друга, и их размеры очень малы по вселенским масштабам. Поэтому весь или практически весь путь космических лучей пролегает по межгалактическому пространству.

Глаз, не вооруженный приборами, воспринимает межгалактическое пространство как абсолютно пустое и темное. Но это не так. В межгалактическом пространстве летят фотоны разных энергий. Одни из них излучены звездами в галактиках. Другие остались от времен рождения Вселенной, и эти фотоны так и называются реликтовые. (Есть в межгалактическом пространстве молекулы газа и пыли и магнитные поля, но о них мы рассказывать не будем.) Космические частицы взаимодействуют с фотонами, и это приводит к двум эффектам.

Первый эффект - это изменение исходной формы спектра космических лучей, а именно: в спектре у Земли по сравнению с исходным спектром не достает частиц самых высоких энергий, около $10^{20}$ эВ и выше. Происходит это по следующей причине.

Когда космические лучи самых высоких энергий летят на Землю с достаточно боль- 
ших расстояний, они на длинном пути успевают взаимодействовать с реликтовыми фотонами. В этих взаимодействиях космические лучи теряют энергию на рождение других элементарных частиц. А космические лучи меньших энергий практически не взаимодействуют с реликтовыми фотонами, так как их энергии недостаточно для производства других частиц. Потратив часть энергии во Вселенной, частицы ультравысоких энергий переходят в ряды космических лучей с меньшими энергиями. В результате их энергетический спектр изменяется по сравнению с исходным спектром.

Значит, чтобы ответить на вопрос, где вблизи сверхмассивной черной дыры ускоряются частицы, недостаточно сопоставлять измеренный спектр космических лучей с теоретическими исходными спектрами.

Но пролет космических лучей по межгалактическому пространству приводит к еще одному эффекту. Он состоит в следующем. Элементарные частицы, которые рождаются во взаимодействиях частиц с реликтовыми фотонами, живут недолго и дают начало электронам, позитронам и квантам. Они в свою очередь взаимодействуют с фотонами, порождая новые и новые электроны, позитроны и кванты. Число частиц растет лавинообразно, и в космосе возникает гигантский каскад из частиц, которые продолжают взаимодействовать с межгалактическими фотонами. Частицы в каскаде расходятся настолько далеко друг от друга, что расстояние между ними превышает размеры Солнечной системы. (Рожденные элементарные частицы дают начало и нейтрино, но они летят по Вселенной, не взаимодействуя.)

Рождение межгалактических каскадов это второй эффект, появляющийся в результате взаимодействий космических лучей с фотонами в космосе.

Для нашего исследования наибольший интерес представляют каскадные кванты. Кроме них в межгалактическом пространстве существуют фотоны различных энергий, и все эти фотоны и кванты составляют внегалактический фон. Его измеряют приборами, размещенными на борту спутников. Основная часть внегалактического фона - это излучение отдельных источников, которые находятся так далеко от нас или же так слабы, что не видны приборами. Возможно, что во внегалактический фон вносят вклад и другие процессы. Например, частицы темной материи, распадаясь, тоже дают начало квантам. Исследуя внегалактический фон, можно определить его компоненты и долю в нем каскадного излучения.

Оказывается, что доля каскадного излучения зависит от того, в какой зоне сверхмассивной черной дыры ускорялись космические лучи. А зная и каскадное излучение, и спектр космических лучей на Земле, можно выяснить, где и как были ускорены частицы. (Напомним, что в каждой зоне они ускоряются своим способом.)

Теперь физики исследуют частицы ультравысоких энергий на Земле и в космосе. Спектр космических лучей измеряют на Земле, а внегалактический фон - в космосе. Анализируя данные этих измерений, физики исследуют процессы в окрестности сверхмассивных черных дыр.

\section{Заключение}

Космические лучи были открыты более ста лет назад, в 1910-х годах. Много лет ушло на понимание взаимодействий космических лучей с земной атмосферой, понимание того, что частицы разных энергий имеют разное происхождение: космические лучи низких энергий выбрасываются Солнцем, более энергичные рождаются в нашей Галактике.

Ученые предполагают, что источники космических лучей ультравысоких энергий - это активные ядра галактик. В их центрах находятся сверхмассивные черные дыры, в окрестности которых частицы приобретают огромную энергию.

Исследование этих источников космических лучей требует создания новых приборов для измерений в космосе. Оно требует понимания, какие процессы возмож- 
ны в активных ядрах галактик и в межгалактическом пространстве. Для моделирования этих процессов требуются усовершенствованные компьютеры.

Мы не сможем попасть в окрестность сверхмассивной черной дыры и непосредственно исследовать протекающие там процессы. Уточнить понимание того, что там происходит, помогает изучение космических лучей и внегалактического излучения, компьютерное моделирование того, как ускоряются частицы и как они летят в межгалактическом пространстве, и сопоставление полученных результатов.

\section{Приложения}

\section{Магнитное поле аккреционного диска}

Межзвездный газ пронизан магнитным полем, оно присутствует во всех звездах. Почти все вещество в звездах и в межзвездной среде ионизовано и состоит преимущественно из заряженных частиц. Вследствие этого в космосе магнитное поле вморожено в среду: силовая линия магнитного поля как бы прикреплена к тем заряженным частицам, которые находились на ней в начальный момент, и когда частицы перемещаются, они увлекают линию за собой.

Газ, падающий на черную дыру и формирующий аккреционный диск, увлекает за собой магнитное поле, поэтому диск намагничивается. А если плотность газа возрастает, т.е. газ сжимается, то силовые линии сгущаются и магнитное поле усиливается. Поэтому поле аккреционного диска может быть намного больше, чем поле, пронизывающее межзвездный газ и вещество звезд.

\section{Способы ускорения частиц вблизи сверхмассивных черных дыр}

Из школьного курса физики известно, что заряженные частицы приобретают энергию в электрическом поле. (Простейший случай: частица с зарядом $q$, пройдя расстояние $L$ в однородном электростатическом поле с напряженностью $E$, приобрета- ет энергию $\delta=q E L$.) Этот механизм ускорения работает и в космосе. Магнитное поле в аккреционном диске неоднородно из-за неоднородной плотности газа. Диск вращается, и его магнитное поле индуцирует электрические поля как в самом диске, так и в магнитосфере черной дыры. Они и ускоряют заряженные частицы. При некоторых условиях индуцированное электрическое поле может ускорять частицы до ультравысоких энергий.

Еще один механизм ускорения частиц в космосе связан с неоднородностями магнитного поля, и в нем не участвует электрическое поле. Впервые возможность такого ускорения частиц понял и рассмотрел итальянский физик Ферми, поэтому этот механизм называется механизмом Ферми. Вблизи сверхмассивных черных дыр механизм Ферми работает в джете, который вырывается из аккреционного диска. Частицы в джете ускоряются так.

Джет сформирован из вещества аккреционного диска. В джете сгустки плазмы выбрасываются из диска по двум воронкообразным каналам вдоль его оси вращения. Толщина аккреционных дисков вокруг сверхмассивных черных дыр неодинакова, она зависит от характеристик черной дыры. В зависимости от толщины диска сгустки летят сквозь него месяцы и годы. Все это время сгустки взаимодействуют с боками канала (с веществом диска) и с излучением диска (ведь аккреционный диск ярко светится в разных диапазонах, он наполнен тепловыми, оптическими, радио- и другими фотонами). В результате взаимодействия с веществом и излучением на поверхности сгустка возбуждаются волны разных типов, в том числе ударные волны. Поясним, что это такое.

Участок поверхности сгустка испытывает удар и получает толчок. Под действием толчка участок плазмы начинает двигаться по сгустку, приводя в движение лежащие впереди плазменные слои. Граница между плазмой, движущейся вдоль сгустка, и неподвижной относительно сгустка резкая. Она называется фронтом ударной 
волны. На этой границе скачком возрастают плотность, давление, температура и скорость плазмы. Ударная волна заставляет плазму в сгустке упорядоченно двигаться, а на фронте происходит интенсивное превращение энергии упорядоченного движения плазмы в энергию хаотического движения частиц. И если частица оказалась на фронте ударной волны, она приобретает энергию, т.е. ускоряется.

Что происходит дальше? Частица уносится потоком плазмы от фронта ударной волны «вниз по течению». Если частица вернется на фронт ударной волны, ее энергия увеличится еще больше. А может ли частица возвратиться? Да, и даже не один раз. Происходит это так.

В сгусток плазмы вморожено магнитное поле, оно неоднородно из-за неоднородной плотности сгустка. В некоторых областях неоднородное магнитное поле направлено так, что отклоняет летящую частицу по направлению к фронту ударной волны, и частица возвращается на фронт. Отклонение частицы пропорционально величине поля и обратно пропорционально энергии частицы - чем она больше, тем слабее отклонение. Поэтому частица возвращается на фронт ударной волны до тех

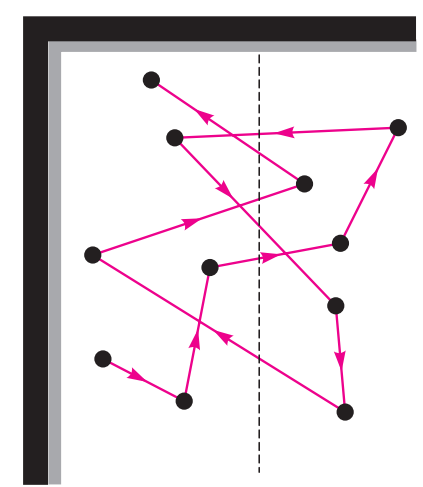

Рис. 3. Блуждания частицы вблизи фронта ударной волны. Фронт показан вертикальной пунктирной линией, частица изображена черным кружком, линии со стрелками - это векторы скорости частицы. Отклонения в неоднородном магнитном поле дают возможность частице многократно пересекать ударный фронт и ускоряться пор, пока не наберет такую энергию, когда магнитные поля в сгустке уже не отклонят ее назад. Движение частицы в неоднородном магнитном поле вблизи фронта ударной волны схематически показано на рисунке 3.

При любом из этих способов ускорения частица одновременно и набирает, и теряет энергию. Энергетические потери возникают, когда частица находится в области, пронизанной магнитным полем с искривленными силовыми линиями. Дело в том, что если силовая линия изгибается, то у частицы появляется составляющая скорости, перпендикулярная магнитному полю. И тогда траектория частицы искривляется под действием силы Лоренца. Частица, которая движется по искривленной траектории, излучает энергию, и энергия самой частицы уменьшается.

Силовые линии могут быть прямолинейными, но лишь на ограниченных отрезках. И даже если частица двигалась вдоль прямой силовой линии, потери энергии все равно будут, и вот почему. Участок, где линия прямолинейна, кончается, и дальше силовая линия изгибается. А в поле с такими силовыми линиями траектория частицы искривляется, частица, двигаясь, излучает энергию и ее энергия падает.

Вблизи сверхмассивных черных дыр частицы все-таки ускоряются до ультравысоких энергий. Это показал теоретический анализ процессов, в которых частица одновременно набирает и теряет энергию.

Оба способа ускорения частиц вблизи черной дыры - посредством индуцированного электрического поля и посредством механизма Ферми - были выявлены теоретически, «на бумаге». Какой из них важнее в природе - покажут наблюдения. 